\title{
ASPECTOS PSICOSOCIALES DEL MANEJO DE LA DISFUNCIÓN ERÉCTIL. HÁBITOS TÓXICOS Y ESTILO DE VIDA. LA PAREJA EN LA DISFUNCIÓN ERÉCTIL. PSICOTERAPIA Y TERAPIA DE PAREJA
}

\author{
Francisco Cabello Santamaría
}

Instituto Andaluz de Sexología y Psicología. España.

\begin{abstract}
Resumen.- OBJETIVO: La sexualidad en general y la respuesta eréctil en particular no son ajenas a aspectos psico-socio-culturales y de pareja.

Poner de relieve aquellos aspectos psicológicos y de pareja que interfieren en la erección y asimismo señalar las pautas de intervención en terapia de pareja que mejores resultados obtienen en el tratamiento de la disfunción eréctil.

MÉTODOS: Se ha hecho una revisión de la literatura en torno a los aspectos psicológicos y sociales según los hábitos de vida actual en relación a la fisiología de la respuesta eréctil, además de revisar los distintos modelos de tratamiento en pareja.
\end{abstract}

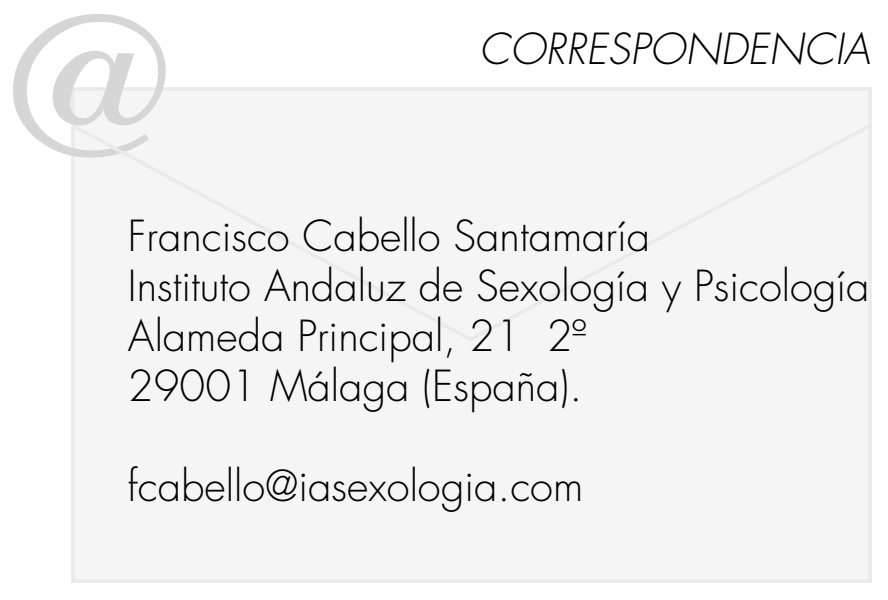

RESULTADOS/CONCLUSIONES: La separación del binomio reproducción-placer conseguido en los años 50 ha permitido una reivindicación progresiva por parte de la mujer de su necesidad de ser satisfecha, constituyendo uno de los motivos que han dado lugar al fenómeno de la "ansiedad de ejecución, que junto con otros hábitos tóxicos (tabaco, alcohol, drogas, vida sedentaria, etc.) facilitan la aparición de disfunción eréctil. Cuando los factores psicológicos forman parte importante del problema, la terapia sexual de pareja combinada con inhibidores de la fosfodiesterasa cinco constituye la estrategia terapéutica de elección.

Palabras clave: Disfunción eréctil. Ansiedad de ejecución. Terapia sexual. Tabaco. Alcohol. IPDE5.

Summary.- OBJECTIVES: Sexuality on the whole and erectile response particularly are not alien to psychosocial and couple features. We review al psychological and couple features that interfere on erection and also point out the interventional directions of couple therapy that give the best results in the treatment of erectile dysfunction.

METHODS: We performed a bibliographic review about the psychological and social features under the current way of life in relation to erectile response physiology, in addition to review different models of couple therapy.

RESULTS/CONCLUSIONS: The separation of the couple reproduction-pleasure achieved in the 50's enabled a progressive claim from women of their need to be satisfied, representing one of the reasons that led to the phenomenon of "performance anxiety" that, altogether with some toxic habits (Tobacco consumption, alcohol, drugs, sedentary life, etc) help the appearance 
of erectile dysfunction. When psychological factors are an important part of the problem, couple sexual therapy in combination with phosphodiesterase 5 inhibitors is the therapy of choice.

Keywords: Erectile dysfunction. Performance anxiety. Sexual therapy. Tobacco. Alcohol. PDE 5 inhibitors.

\section{HABITOS TÓXICOS Y ESTILO DE VIDA}

La erección tiene una importancia capital en todas las culturas y épocas, habiéndose exaltado en pinturas prehistóricas, definido en papiros egipcios y deificado, como es el caso del dios Príapo, pero hasta mediados del siglo XX la ausencia de erección no tenía una repercusión social, ni relación con el éxito personal simplemente era una enfermedad -impotencia coeundi- que mostraba importancia en el campo de la fertilidad.

La secularización de la sociedad, definitivamente asentada durante la centuria pasada, la conquista de libertades civiles, la emancipación de la mujer y la aparición de una anticoncepción eficaz (la "píldora" aparece en los años 50), permitieron separar la reproducción del placer y con ello una reivindicación progresiva por parte de la mujer de su necesidad de ser satisfecha, cuestión que no había tenido relevancia en épocas anteriores.

Mucho debió contribuir a este asunto la publicación en 1953 del informe Kinsey (1) sobre sexualidad femenina, que constituyó el salto a la luz, de forma empírica, de las demandas de las mujeres en materia sexual.

En este contexto el hombre, que en otros momentos históricos sólo estaba preocupado por su satisfacción, asumió la responsabilidad del placer femenino naciendo de esta manera la "ansiedad de ejecución" fenómeno que influye de manera notable en la repuesta eréctil.

Esta ansiedad es desencadenada por lo que Abraham y Porto denominaron factores generadores de ansiedad (2), a saber:

1. Temor al fracaso (sensación de miedo a no responder de forma adecuada ante la pareja).

2. Obligación de resultados (necesidad de una respuesta eréctil muy consistente, duradera y de recuperación rápida).
3. Altruismo excesivo (estar más pendiente de la satisfacción de la pareja, perdiendo concentración en el erotismo propio).

4. Autoobservación, consistente en estar observando el pene para ver cómo responde, lo cual había sido denominado previamente "rol de espectador" (3).

En nuestro entorno cultural el norte es el éxito, basado en la cantidad de dinero, posesiones, posibilidades de consumo, etc., y extendido al campo de las relaciones afectivas donde lo más importante radica en tener habilidades de seducción, un enorme tamaño de pene, una erección consistente y un tiempo eyaculatorio que permita la multiorgasmia. Todo ello termina convirtiendo las relaciones sexuales en una carrera de obstáculos en pro de a la eficacia en vez de una situación placentera para compartir la intimidad. Por tanto, al estrés vital resultante de la lucha por conseguir la máxima eficacia en el terreno laboral y social se le suma el estrés sexual, que desde un punto de vista fisiológico en nada ayuda a la respuesta eréctil.

Es bien sabido que el tono adrenérgico elevado propio de las situaciones estresantes inhibe la erección y cómo este estrés continuado facilita la producción de prolactina y por tanto favorece la disminución del deseo.

Al mismo tiempo, como mecanismo mitigador de los altos niveles de ansiedad propios del estilo de vida de nuestro medio, se incurre en determinados hábitos tóxicos, es decir, los tiempos de descanso que debieran ser dedicados a actividades de deportivas de carácter ocioso y al descanso intelectual, comúnmente se emplean en celebraciones culinarias nada saludables, acompañadas de la degustación, de forma excesiva, de vinos y licores además del consumo de tabaco, cuando no del uso y abuso de drogas ilegales (cocaína, cannabis, etc.). Por ejemplo, cada cigarrillo produce, por el efecto de la nicotina, una media hora de vasoespasmo arterial, por lo cual, si alguien está despierto unas 16 horas por día y fuma 30 cigarrillos diarios no le dará descanso a sus arterias, que se mantendrán en contracción casi todo el día, lo que no es una ayuda para los vasos del pene que necesitan dilatarse para lograr la erección. Además para el mantenimiento del endotelio peneano son fundamentales las erecciones nocturnas y se ha demostrado que la rigidez de la erección durante el sueño correlaciona inversamente con el número de cigarrillos fumados por día (4).

Respecto al alcohol, que en el medio que habitamos se encuentra estrechamente ligado al sexo, es muy frecuente iniciar un encuentro sexual justo des- 
pués de haber tomado unas copas y si bien, concentraciones muy bajas de alcohol en sangre tienen un suave efecto intensificador del deseo y la erección, concentraciones correspondientes a dos o tres copas de licor disminuyen la fuerza eréctil y retardan la eyaculación (5).

El alcohol actúa a dos niveles: por un lado produce neuropatía y alteraciones de la neurotransmisión $y$, por otro, desequilibrios hormonales. El etanol y el acetaldehído, componentes básicos de todas las bebidas alcohólicas, producen un efecto tóxico sobre las células de Leyding inhibiendo las enzimas responsables de la elaboración de hormonas. El eje hipotalámico-hipofisario también se bloquea por el efecto directo del etanol y el aumento de la concentración de estrógenos en sangre (6). A consecuencia de ello aparecerá atrofia testicular, bajos niveles de testosterona y disminución de la espermatogénesis (7). Además con el paso del tiempo la ingesta de alcohol propicia una disminución de las neuronas hipotalámicas responsables de la producción de oxitocina, lo que tiene mucho que ver con la respuesta orgásmica masculina, de ahí la alta frecuencia de anorgasmia entre los bebedores crónicos.

No obstante, se podría considerar que el principal hábito tóxico que terminará a largo plazo interfiriendo con la erección lo constituye la vida sedentaria y la alimentación desequilibrada.

\section{LA PAREJA EN LA DISFUNCIÓN ERÉCTIL}

El habitual esquema de comunicación de los hombres es distinto al de las mujeres (9). Los primeros, cuando tienen algún problema suelen omitirlo hasta que no encuentran una solución. Además, el varón por lo general no sólo no habla del asunto para aclarar la situación, sino que huye, se centra más en el trabajo, amigos o actividades deportivas, intentando estar el menor tiempo posible a solas en compañía de su pareja.

Otro factor muy común, que agrava el ambiente de incomunicación y el descontento de la pareja, se debe a la poca consideración del paciente con disfunción eréctil, que en el momento que obtiene una erección casual, rápidamente intenta penetrar sin tan siquiera estimular a la pareja por temor a perder la firmeza en el proceso. También es corriente despertar a la mujer de madrugada porque se ha notado el pene erecto y aprovechar la ocasión. Estas conductas no son interpretadas por la mujer como prisa por conseguir penetrar y poder satisfacerla, sino como una prueba de egoísmo.
Ante esta situación las mujeres se hacen las siguientes preguntas (10):

- ¿Es por mi culpa?

- ¿́Soy poco atractiva y por eso no se excita?

- ¿Habrá otra persona?

- ¿Habré estado demasiado centrada en los hijos o en el trabajo?

Todas esas dudas van generando un alto nivel de frustración en la mujer y cuestionándole su relación de pareja, creando en el hombre una alta demanda de ejecución.

Según Barlow, la demanda de relaciones por parte de la pareja de la persona con disfunción eréctil, hace que el individuo reaccione muy negativamente (11). Siguiendo a este autor el proceso sería el siguiente: la demanda de ejecución por parte de la pareja, provoca que el individuo genere unas actitudes negativas hacia la relación con percepción de falta de control. El sujeto estaría concentrado en las consecuencias de su imposibilidad para penetrar, lo que aumentaría su ansiedad, conllevando una pérdida o ausencia de erección y por tanto tendencia a la evitación sexual. Por el contrario, quienes disfrutan experiencias sexuales positivas, ante la demanda de relaciones sexuales por parte de la pareja, cabe esperar que tengan expectativas de correcta erección, por tanto una predisposición positiva, la atención centrada en las sensaciones eróticas, una respuesta adecuada $y$, como consecuencia, tendencia al acercamiento (Tabla I).

Partiendo de este esquema, la demanda de ejecución junto con las dudas sobre la respuesta eréctil, harán que el individuo aumente su ansiedad y bloquee la erección. El mecanismo, se vuelve con el tiempo cada vez más perverso, ya que los pacientes con disfunción eréctil tienden, como se ha señalado anteriormente, a la evitación de los momentos sexuales para no caer de nuevo en la frustración consecuente al fracaso y con la idea de no incitar a la pareja para terminar dejándola insatisfecha.

Ante esta situación, la pareja nunca va a responder de forma neutra, variando con respecto a su propia respuesta sexual, así podemos diferenciar entre tres posibilidades (12):

\section{Mujeres con un buen nivel de satisfacción sexual.}

2. Mujeres con una sexualidad poco activa sin disfunción sexual.

3. Mujeres que presentan alguna disfunción sexual. 


\section{Sujetos con buen} funcionamiento

\author{
Percepción de control<smiles>[3H][3H]</smiles> \\ Atención centrada en \\ sensaciones eróticas \\ Excitación y abandono<smiles>[3H][3H]</smiles> \\ Aumentando la atención \\ en lo erótico \\ Ejecución correcta \\ Tendencia al acercamiento
}

\section{Sujetos con disfunción Feedback negativo}

\section{Demandas de ejecución}

Percepción de falta de control

Atención centrada en las consecuencias públicas de la no ejecución

Aumento del "arousal" autonómico o sea la ansiedad somática

Que a su vez aumenta la atención centrada en consecuencias de la no ejecución

Ejecución incorrecta<smiles></smiles>

Tendencia a la evitación
Las primeras presentan conductas demandantes de mayor intensidad, presionando constantemente a la pareja y obligándoles para que busquen alguna solución al problema. En esos casos el paciente se ve atosigado, aumenta su ansiedad y consecuentemente sus conductas evitantes, escudándose en argumentos tales como "que la sexualidad no es lo más importante de una relación, "que su pareja está obsesionada con el sexo", etc.

La mujer disfuncional (anorgasmia, deseo sexual hipoactivo, aversión sexual, etc.) por el contrario, suele reaccionar de dos formas bien distintas. Algunas suele animar al paciente con disfunción eréctil en el sentido de quitarle importancia al problema, se muestran comprensivas y convencen al sujeto de que su problema no es grave argumentando que la sexualidad es poco importante dentro de la relación de pareja. Otras achacan su propia disfunción al trastorno de su pareja, fenómeno especialmente común entre mujeres anorgásmicas con facilidad para la excitabilidad.

Por último, quienes presentan una sexualidad poco activa, suelen reaccionar de forma parecida a algunas mujeres disfuncionales, evitando la sexualidad y convenciéndose a sí mismas de la poca importancia del sexo en sus vidas.

En suma, en esta disfunción sexual más que en cualquier otra, la pareja se ve afectada interactuando en el proceso, de forma que un $40 \%$ de las mujeres terminan presentando una disfunción sexual comórbida al trastorno eréctil (13).

\section{PSICOTERAPIA Y TERAPIA DE PAREJA}

Según los estudios de diversos autores $(14,15)$, hasta la aparición de los IPDE5, la eficacia de la terapia sexual había sido superior al resto de las intervenciones (fármacos u otros abordajes). En el caso concreto de la disfunción eréctil secundaria, el número de fracasos terapéuticos es de sólo un $26^{\prime} 2 \%$ (3) y, tal como afirma McConhagy, la terapia sexual es muy eficaz, debiéndose los fracasos terapéuticos, en su mayor parte, a la falta de motivación y abandono del tratamiento (16).

La intervención está conformada por un conjunto de estrategias de carácter psicoterapéutico que se emplean con los siguientes fines: 
- Tratamiento de la disfunción eréctil de carácter psicógeno.

- Optimización del tratamiento con fármacos en la disfunción eréctil de origen orgánico.

- Rehabilitación de la respuesta sexual en aquellos casos donde el tratamiento farmacológico no surte efecto.

- Adaptación y optimización de la respuesta en enfermedades crónicas que imposibilitan la erección.

Los modelos empleados con mayor frecuencia para el tratamiento de la disfunción eréctil se citan a continuación.

\section{Modelo terapéutico de Masters y Johnson}

La terapia sexual que proponen Masters y Johnson (1970) (3), que es llevada a cabo por dos terapeutas (hombre y mujer) al mismo tiempo, parte de la prohibición del coito para iniciar la denominada por ellos focalización sensorial, consistente en que la pareja desnuda comienza a masajearse sin pasar a caricias genitales en un primer tiempo y después incluyendo los genitales.

Una vez conseguida la erección se procede a:

- "Técnica fastidiosa": radica en manipular el pene hasta conseguir la erección y después, interrumpir el estímulo para permitir la distracción y pérdida de la erección, retomando de nuevo la manipulación del pene. Esta estrategia se debe mantener durante media hora.

- Una semana más tarde, cuando el pene entra en erección tras la estimulación, la mujer debe excitar los genitales sentada en cabalgada sobre el hombre.

- Cuando ella se encuentre cómoda en esa postura, debe introducir el pene en su vagina, con lo cual se elimina la natural distracción erótica del varón al intentar buscar el orificio vaginal.

- La introducción del pene por parte de la mujer se repite en varias ocasiones hasta que el varón se siente seguro. Si no hubiera erección se sigue con el juego erótico sin prisas.

- A continuación ella comienza a moverse lentamente intentando evitar cualquier tipo de demanda sexual.

A partir de ahí, se continuarán, en los días siguientes, los ejercicios de los "placeres pelvianos". Se basa en la repetición del paso anterior, sin preten- der la satisfacción de la mujer ni la eyaculación del hombre, hasta que se vaya normalizando la situación.

\section{Modelo terapéutico de Helen Singer Kaplan}

El modelo de Kaplan (17) parte de las propuestas de Masters y Johnson (3), pero con una sistemática más flexible, adaptada a cada persona, empleando procedimientos que no se hallan organizados de forma rutinaria y partiendo de las necesidades psicodinámicas de la pareja.

Comienza con la focalización sensorial, siendo el objetivo fundamental del terapeuta conseguir una primera erección que va a servir de referencia al paciente de su capacidad para luchar contra los bloqueos que tiene. Esta primera erección se conseguirá a partir de:

- La focalización sensorial, haciendo énfasis en la abstinencia inicial del orgasmo.

- El empleo provisional de fantasías para sustraerse de la ansiedad.

La autora, pese a ser psicoanalista, carga de eclecticismo lo que ella denominaría "nueva terapia sexual" y recubre el modelo con la ideología psiquiátrica de la época, basando en última instancia la resolución de los problemas en el "insight".

\section{Modelo terapéutico de Gérard Zwang}

Para Zwang (18) la terapia sexual estaría centrada en el síntoma, que tiende a recondicionar favorablemente a hombres y mujeres sanos física y psíquicamente, pero con una psicología erótica inadecuada. Para ello se debe prohibir cualquier ocasión de repetición del fracaso, es decir:

- Descondicionar a la pareja de su estilo erótico desfavorable.

- Eliminar la necesidad imperiosa de obtener el orgasmo.

Se debe informar y desculpabilizar mediante una guía de instrucciones impresas y generar un ambiente para mantener o restaurar el deseo sexual. En este contexto se procederá a:

- Estimulación corporal extragenital con prohibición de acariciar el pene.

- Estimulación del hombre delante de su pareja hasta obtener la erección para familiarizarse con la situación. 
- Pasar a la penetración en posturas laterales o en cabalgada.

\section{Modelo terapéutico de Keith Hawton}

Este autor (15) se basa inicialmente en las proposiciones introducidas por Masters y Johnson (3) y desarrolladas por Kaplan (17). El modelo consta de las siguientes etapas:

- Focalización sensorial no genital. Donde, un miembro de la pareja, cuando le apetezca, debe invitar al otro a realizar los ejercicios en casa. Después de la primera sesión de caricias, el patrón de invitaciones es alternante, de manera que la responsabilidad de la invitación recaerá sobre el otro miembro en la sesión siguiente.

- La focalización sensorial genital sería el segundo paso, donde el autor emplea la técnica del "aumento y la disminución del estímulo". Durante una sesión, una vez que el varón ha tenido una erección intensa se debe aconsejar a la pareja que cese todo contacto físico, permitiendo que la erección disminuya. Entonces deben reiniciar las caricias, acariciando la mujer con suavidad el pene del hombre, quien deberá recuperar la erección. El procedimiento debe repetirse 2 o 3 veces en cada sesión.

- Contención vaginal, donde la penetración debe llevarse a cabo con la mujer guiando el pene de su pareja con las manos. Una vez producida la penetración vaginal, la pareja debe permanecer inmóvil y concentrada en las sensaciones placenteras que experimentan. El hombre puede moverse ligeramente para estimularse si su erección comienza a disminuir y emplear fantasías sexuales; al mismo tiempo, la mujer debe contraer los músculos de su vagina.

- Contención vaginal con movimiento. En esta etapa se introduce movimiento durante la contención vaginal y progresivamente se avanza hasta llevar a cabo una relación coital natural. Se recomienda a la mujer que comience el movimiento después el hombre. A continuación establecerán turnos en el movimiento.

\section{Biblioterapia y autoayuda}

Ha sido frecuente en el campo de la terapia sexual la existencia de materiales de autoayuda, posiblemente porque la gente ha tenido $y$, aunque con algunos cambios, sigue teniendo dificultades para consultar sobre sus problemas sexuales.

De entre los programas de autoayuda referidos a la disfunción eréctil, son destacables el pro- puesto por Carole Altman (19) y la adaptación de Masters y Johnson (5) de su esquema terapéutico.

\section{Modelo de Carole Altman}

Comienza por una autoevaluación a partir de un cuestionario que determina si la causa de la disfunción es probablemente de carácter psicológico, o por el contrario, de base orgánica; en cuyo caso, se advierte de la poca probabilidad de mejoría siguiendo el programa de autoayuda.

La filosofía del programa se nutre de la idea de que, al igual que en otros campos, las personas que trabajan y se esfuerzan por conseguir una vida sexual agradable y excitante tienen muchas más probabilidades de conseguirla que las que ignoran su sexualidad. Este esfuerzo se va a ver reflejado en una serie de contratos con la pareja que son imprescindibles y de obligado cumplimiento.

a) "Contrato I: Contrato de la no exigencia sexual" La persona que siente la necesidad de una mejora sexual no debe experimentar durante la terapia ninguna sensación de fracaso o de presión; por tanto, los componentes de la pareja deben evitar toda actividad sexual.

b) "Contrato II: Vamos a intentarlo de verdad"

Este contrato debe entrar en vigencia a la vez que el anterior y debe llegar a un acuerdo sobre las tareas, el horario, la forma de llevarlas a cabo, etc.

c) "Contrato III: contrato de no penetración" Es el consentimiento por ambas partes de tener actividad sexual sin pasar a la penetración.

d) "Contrato IV: contrato de la actividad sexual plena"

Una vez que mediante los ejercicios anteriores se ha ganado confianza y se consiguen erecciones ante diferentes estímulos, se procede al contrato de la actividad sexual plena, dejando siempre en mente la posibilidad de algún fracaso ocasional ("retirada", en palabras de Altman) que se ha dejado redactada por escrito con antelación.

\section{Modelo de autoayuda de Masters, Johnson y Kolo- dny}

Partiendo de los principios generales de su modelo, Masters, Johnson y Kolodny (5), fieles a su filosofía primigenia, dividen su estrategia terapéutica en pequeños pasos, en un lenguaje fácilmente entendible y, a diferencia de la terapia inicial, dedican un importante peso a la realización de fantasías durante la realización de las tareas. 
En el Instituto Andaluz de Sexología y Psicología, partiendo de los modelos citados, se han ido modificando algunas estrategias e integrando nuevas herramientas adquiridas por la experiencia con los pacientes, hasta desarrollar un modelo propio que consta de dos niveles: simple y combinado.

\section{Modelo del Instituto Andaluz de Sexología y Psico- logía}

Dicho modelo ha sido evaluado de forma empírica, así, en el momento actual se puede afirmar que haciendo uso del modelo simple el $66,7 \%$ de los pacientes superan el problema y si se emplea el modelo combinado la eficacia del tratamiento alcanza el $90 \%(12)$.

\section{Modelo simple}

De forma resumida los pasos serían los siguientes:

\section{a) Erotización sensual}

Prohibición del coito

Desde la primera sesión se aconsejan ejercicios encaminados a disminuir la ansiedad de ejecución. Para ello se prohíbe la realización del coito, tal como establecieran Masters y Johnson (3), que tiene como finalidad hacer desaparecer la ansiedad y la demanda de ejecución.

Revitalización y descubrimiento de mapas eróticos

En caso de que la relación de pareja sea aceptable, se comenzará con el masaje sensitivo, recalcando la necesidad de recorrer todo el cuerpo de forma sensual. Antes de proceder a realizar la erotización hay que tener la seguridad de que existe un nivel de motivación adecuado por parte de ambos miembros de la pareja, pues si se comienzan los ejercicios y no conducen a buen fin, debido a una escasa colaboración, se habrá desperdiciado una herramienta cuya eficacia no es la misma en segundas vueltas.

\section{Autoestimulación}

Se le refiere al paciente que la autoestimulación sirve como rehabilitación del pene, en el sentido de favorecer la vascularización y tonificar la musculatura genital. Antes de aconsejarle que se masturbe, se le habrá explicado con detenimiento el fenómeno de la autoobservación.

Es común que el paciente se masturbe para comprobar si es capaz de obtener la erección. Con ello, al principio, se puede conseguir la erección con normalidad, pero el paso del tiempo va haciendo que disminuya la respuesta y que la misma ansie- dad movilizada ante la pareja se exprese en solitario impidiendo que el pene alcance la dureza deseada. Por tanto, es normal que el individuo argumente que de nada sirve masturbarse porque no consigue erección, a lo que se le debe responder que no es necesario obtenerla, ni tan siquiera que se excite o alcance el orgasmo, ya que el objetivo es tonificar la musculatura y vasos del pene.

\section{Comunicación sexual}

Para obtener buenos resultados es imprescindible una comunicación fluida, al menos, en lo relativo a la sexualidad. Es común en parejas disfuncionales desconocer por completo las preferencias en materia sexual del otro, por eso ya en esta primera fase de la terapia, se recomienda que cada día dediquen un tiempo preestablecido para hablar de sexualidad. En concreto, se dividirá el proceso en tres fases:

1. Expectativas sexuales: Comentarán la sexualidad ideal de cada uno (previamente en consulta se les entrena en unas normas básicas de comunicación).

2. Negociación: En este apartado negociarán las demandas mínimas necesarias para tener una sexualidad satisfactoria por parte de cada miembro de la pareja.

3. Elaboración de fantasías: Una vez realizados los pasos anteriores, se recomienda la elaboración de fantasías sexuales conjuntas y, si les apetece, se les recomienda la construcción de distintos escenarios donde llevar a cabo la práctica de los ejercicios de erotización.

Toda la intervención terapéutica va acompañada de un proceso permanente de educación de la sexualidad y reestructuración cognitiva de las ideas irracionales del paciente y la pareja. Además, en esta fase se hace necesario promover las habilidades de negociación en pareja, ya que si no reina la armonía, difícilmente funcionará la terapia sexual.

\section{b) Erotización genital}

Una vez que la pareja ha superado el paso anterior y el paciente se relaja o bien se excita sin estar demasiado pendiente de la erección, se pasa a la siguiente fase, es decir, a la erotización genital.

\section{Erotización genital concentrándose en el tacto}

La erotización genital incluye acariciar los genitales, pero advirtiendo que las caricias no se harán de forma diferente a como se acaricia el resto del cuerpo y, aunque aparezcan erecciones, se habrá de obviar el coito. Una vez más se le informa de que no esperamos que obtenga una erección, sino que el objetivo consiste en encontrarse cómodo. 
Erotización genital concentrándose en fantasías eróticas

El paciente deberá abstraerse en fantasías eróticas mientras es acariciado. La finalidad de esta estrategia es generar "distracción cognitiva" en el sentido propuesto por Barlow 1 1, para que el paciente no esté pensando durante las caricias "no puedo", "soy incapaz de conseguir una erección", etc.

\section{c) Erotización orgásmica}

Si el paciente está colaborando y no evita las tareas, se pasa a la denominada erotización orgásmica, que en esta ocasión consta de tres fases:

- Caricias compartidas. Tras realizar unos cinco minutos de erotización sensual y genital, se pasa a caricias compartidas a modo de petting con la prohibición del coito.

- Autoestimulación delante de la pareja. Si las tareas anteriores han evolucionado de forma adecuada y el paciente no tiene problemas con la erección cuando se masturba en solitario, es el momento de pedirle que se autoestimule hasta conseguir la erección mientras ella le acaricia cualquier parte del cuerpo. Una vez alcanzada la erección, el paciente debe parar hasta que la erección desaparezca, entonces repetirá de nuevo el proceso en tres ocasiones. Al cuarto intento, si le apetece, puede seguir estimulándose hasta llegar al orgasmo.

- Rozamiento genital. Esta tarea se entremezcla con las anteriores, de forma que a lo largo de los ejercicios deben dedicar unos minutos, cuando hay erección, al rozamiento de los genitales. Tras autoestimularse, el varón rozará sus genitales contra los labios menores y clítoris de la mujer. A continuación, adoptará una posición pasiva y será ella, quien cogiendo el pene, lo roce con sus genitales en cabalgada (se advierte que bajo ningún concepto se debe intentar la penetración). Llegado este momento, se permitirá alcanzar el orgasmo pero sin penetración. Se pretende que el paciente se familiarice con la erección delante de su pareja, acontecimiento que no ocurría anteriormente, sin el miedo de un posible fracaso.

\section{d) Capacitación para el coito}

Partiendo de la erotización orgásmica se debe continuar con la capacitación para el coito, que consta de los siguientes pasos:

- Penetración inmóvil con autoestimulación. Al individuo que obtiene fácilmente la erección por caricias de su pareja o autoestimulación, se le pide que una vez conseguida la erección, su pareja se introduzca el pene en la vagina, estando sentada encima con las rodillas situadas junto al costado, por debajo de los pezones del varón (en cabalgada). Una vez que está el pene dentro, el paciente se queda inmóvil hasta que desaparezca la erección. Llegados a esta fase debe comenzar a autoestimularse de nuevo, compartiendo la masturbación con ella y al reaparecer la erección se procede de nuevo a que ella introduzca el pene en la vagina. Los ejercicios se finalizan con la masturbación del paciente delante de su pareja, dándole la opción de estimularla a ella hasta el orgasmo, - bien que sea ella quien se estimule a sí misma. Con esta tarea se pretende habituar al paciente a que pierda la erección sin miedo y, además, hacerlo consciente de que la puede recuperar por sí mismo.

- Estrategia del "cartero". Denominada así porque el autor hace referencia cuando explica esta tarea a sus pacientes, a una famosa escena erótica de la película "El cartero siempre llama dos veces" (dirigida por Bab Rafelson en 1981). Se trata de que el paciente se autoestimule hasta alcanzar la erección mientras ella se encuentra en decúbito supino al borde de una superficie alta, por ejemplo una mesa, o bien puede estar al borde de la cama, mientras él se apoya con las rodillas en el suelo. En esa situación él está masturbándose en la cercanía de los genitales de ella. Cuando tiene erección se roza $y$, sin dejar de frotarse, introduce el pene. Una vez dentro lo retira de inmediato para seguir autoestimulándose.

Una vez alcanzada esta fase con éxito el pronóstico es muy favorable, sobre todo si la relación de pareja es armoniosa.

\section{e) Coito libre}

Transcurrido un mes practicando con éxito las tareas anteriores, se recomienda acentuar los ejercicios durante la penetración. Se solicita que durante la penetración inmóvil aumenten los empujes intravaginales pero a un ritmo lento. En todo caso el orgasmo no debe producirse con la penetración, esto quiere decir que cuando el placer sea intenso, próximo a la eyaculación, el pene debe ser retirado para alcanzar el orgasmo con caricias y juegos.

Una vez en esta etapa, parece lógico permitir la realización espontánea del coito una vez conseguida la penetración, pero el número de recaídas es mayor con respecto a seguir con pautas terapéuticas (muy parecidas de hecho a una relación natural), que le evitan al paciente responsabilidad y demanda de ejecución, lo que permite que afiance los hábitos conseguidos. Así, salvo pacientes con personalidad obsesiva, fieles cumplidores de las prescripciones, es 
habitual que en mitad de esta fase, muchas parejas dejen de respetar las propuestas y de forma natural vayan acoplándose a una relación saludable. A otras, tras un mes de realizar estas prácticas, se les da permiso para que procedan a una relación espontánea, pero practicando la penetración inmóvil al menos una vez cada dos semanas durante seis meses.

\section{Modelo combinado}

Desde la práctica clínica se entiende que en toda disfunción eréctil, independientemente de la causa originaria, como factor mantenedor de la disfunción se va a encontrar un fuerte componente psicológico que puede dificultar el tratamiento.

De la misma manera se tiene constancia de que cuando la pareja no colabora o no se siente integrada en el proceso terapéutico, la adherencia al tratamiento farmacológico disminuye, siendo frecuentes los abandonos de la medicación. Incluso tratamientos farmacológicos efectivos para la erección como las inyecciones intracavernosas, son abandonados por el paciente cuando la pareja no colabora $(20,21)$. Por tanto, el tratamiento de la disfunción eréctil debe hacerse siempre que sea posible en pareja, lo que favorecerá una respuesta más adecuada además de evitar futuros abandonos con independencia de la etiología de la disfunción.

En cualquier caso, la razón fundamental para la utilización de un tratamiento combinado (fármacos + terapia sexual) parte de la ansiedad de ejecución que experimenta el paciente con disfunción eréctil (miedo al fracaso, etc.), que en ocasiones conlleva una desaparición del deseo, imprescindible para que funcionen los inhibidores de la fosfodiesterasa. Además la ansiedad aumenta el tono adrenérgico, con lo cual el flujo sanguíneo en el pene decrece.

Según demuestra el modelo de Barlow (11) citado con anterioridad, uno de los elementos generadores de ansiedad anticipatoria (miedo al fracaso) es lo que este autor denomina "demanda de ejecución" por parte de la pareja. Esto significa que cuando aparece una disfunción eréctil, la pareja va a presionar para que el paciente ejecute una respuesta sexual adecuada y esta exigencia propiciará un mayor nivel de ansiedad. Por tanto, cuando la pareja colabora disminuye la "demanda de ejecución" (aspecto fundamental sobre el que se interviene en terapia sexual), elemento facilitador de la respuesta sexual y, de ahí, que la terapia sexual en pareja, combinada con inhibidores de la fosfodiesterasa, pueda incrementar la eficacia del fármaco, así como el fármaco puede incrementar la eficacia de la terapia sexual.
El tratamiento de elección para la disfunción eréctil, según nuestro criterio, consiste en el empleo del modelo previamente descrito junto con los IPDE5, tal como sigue:

a) Erotización sensual. Se llevará a cabo tal como se ha detallado en el apartado de terapia sexual, es decir, en esta fase no se emplea la medicación.

b) Erotización genital con fármacos. En esta fase, especialmente en pacientes de larga evolución y también en aquellos con una relación de pareja conflictiva, se añade un IPDE5. Se remarca la prohibición del coito, con el objetivo de familiarizar a la pareja con las erecciones en un proceso de comunicación y erotización.

c) Erotización orgásmica con fármacos. Los ejercicios se realizarán de igual manera que en el modelo descrito, pero tomando IPDE5. El paciente debe realizar las tareas correspondientes, es decir, caricias compartidas, autoestimulación delante de la pareja y rozamiento genital. Se le permitirá alcanzar el orgasmo pero sin penetración. De esta forma el paciente se familiariza con la erección delante de su pareja y toma confianza. Por otro lado, se asume que la disminución de la ansiedad de ejecución, al no tener que enfrentarse al coito, facilitará la excitación y, por tanto, la liberación de óxido nítrico y disminución del tono adrenérgico, permitiendo la actuación del IPDE5.

d) Capacitación para el coito con fármacos. Una vez superados los pasos previos se utiliza la penetración inmóvil. En esta situación se utiliza de forma determinante la intención paradójica, al pedirle al paciente que se mantenga quieto dentro de la vagina hasta perder la erección, para cuando esto ocurra comenzar de nuevo la estimulación. El IPDE5 dificultará la detumescencia y el pene no bajará, experimentando una agradable sensación de control. A continuación se le pide que se mueva con lentitud dentro de la vagina sin permitirle la eyaculación dentro (así no tiene la percepción de estar en una relación coital habitual que le puede desencadenar el miedo al fracaso). Estas tareas se alternan con el empleo de la "estrategia del cartero".

e) Coito libre sin fármacos. Transcurridas siete sesiones de ejercicios se permite el coito libre. Una vez llegado a este punto se intenta la retirada progresiva del fármaco, consiguiéndose buenos resultados en las disfunciones eréctiles de origen psicógeno.

Esta estrategia persigue que el tratamiento tenga un efecto "curativo" definitivo, sin necesidad de tener que seguir usando fármacos para conseguir 
la erección. Obviamente en pacientes con lesiones orgánicas el fármaco seguirá siendo necesario, pero si no se ha llevado a cabo este proceso es probable que las dudas ante la respuesta, la demanda de ejecución y, en suma, el miedo al fracaso, dificulten la acción del medicamento. Además la secuencia de ejercicios facilita el proceso de comunicación y un mejor entendimiento en pareja, facilitando también el cumplimiento del tratamiento.

La terapia combinada ha resultado ser más eficaz que el tratamiento farmacológico y la terapia sexual como estrategias aisladas en el tratamiento de la disfunción eréctil de origen psicógeno. El 90\% de los pacientes siguen con erecciones adecuadas un mes después de haber terminado la intervención terapéutica. Además el nivel de abandono de los distintos tipos de intervención es mucho menor con la terapia combinada (el $23 \%$ de los abandonos corresponden a terapia farmacológica, el $69 \%$ a terapia sexual y el $8 \%$ a la terapia combinada) (12).

\section{CONCLUSIÓN}

En el Instituto Andaluz de Sexología y Psicología, hoy día se inicia el tratamiento de la disfunción eréctil con el modelo de terapia combinada, dejando el modelo simple para aquellos casos que tienen reparos en tomar medicación, disfunciones eréctiles psicógenas de corta evolución y contraindicaciones a los IPDE5.

\section{BIBLIOGRAFÍA Y LECTURAS RECOMENDADAS ( ${ }^{*}$ lectura de interés $y^{* *}$ lectura fundamental)}

1. Kinsey AC, Pomeroy WB, Martín CE, Gebhard, $P$. Sexual Behaviour in the human female. Filadelfia: W.B. Saunders Co; 1953.

2. Abraham G, Porto R. Terapias Sexológicas. Madrid: Pirámide; 1979.

*3. Masters W, Johnson V. Incompatibilidad sexual humana. Buenos Aires: Interamérica; 1970.

4. Hirshkowitz M, Karacan I, Howell JW, Arcasoy MO, Williams RL. Nocturnal penile tumescence in cigarette smokers with erectile dysfunction. Urol 1992; 39(2), 101-107.
5. Masters W, Johnson V, Kolodny R.. Heterosexuality. New York: Harper Collins; 1994.

6. Steenbergen WV. Alcohol, liver cirrhosis and disorders in sex hormone metabolism. Acta Clínica Bélgica 1993; 48(4): 269-83.

7. Taniguchi N, Kaneko S. Alcoholic effect on male sexual function. Nippon Rinsho 1997; 55(11): 3040-4.

8. Silva SM, Madeira MD, Ruela C, Paula-Barbosa MM. (2002). Prolonged alcohol intake leads to irreversible loss of vasopressin and oxytocin neurons in the paraventricular nucleus of the hypothalamus. Brain Research 2002; 925, 76-88.

9. Van Pelt NL. How to talk so your mate will listen and listen so your mate will talk. New York: Fleming H. Revell Company; 1989.

10. Basson R. Succesful management of erectile dysfunction: adressing its repercusions on the female partner. The first European congress on the management of male sexual dysfunction and other critical issues in men's health. Mónaco; 2002 Abr.

11. Barlow DH. Causes of sexual dysfunction: The role of anxiety and cognitive interference. Journal of Consulting and Clinical Psychology 1986; 54: 140-8.

**12. Cabello F. Manual de sexología y terapia sexual. Madrid: Síntesis; 2010.

13. Cabello F. Disfunción eréctil: Un abordaje integral. Psimática: Madrid;2004.

14. Kockott G, Dittmar F, Nusselt L. Systematic desensitization of erectile impotence: a controlled study. Arch Sex Beh 1975; 4(5), 493-500.

*15. Hawton K. Terapia Sexual. Barcelona: Doyma; 1985.

16. Mcconhagy N. Disfunción y Desviación Sexual. En: Bellack AS, Versen M. Manual Práctico de Evaluación de Conducta. Bilbao: Desclée de Brouwer; 1990, 531-75.

17. Kaplan HS. The new sex therapy - Active treatment of sexual dysfunctions. New York: Times Book Co;1974

18. Zwang G. Sexologie. París: Masson;1985.

19. Altman, C. (1978). You can be your own sex therapist. New york: See Hear Productions Inc.

20. Jannini EA, Lenzi A, Wagner G. New perspectives in the pharmacotherapy of erectile dysfunction. IDrugs 2003;6(12), 1165-1172.

21. Pallas J, Levine SB, Althof SE, Risen CB. A study using Viagra in a mental health practice. J Sex Marital Ther 2000; 26(1), 41-50. 